\title{
The American Association for Thoracic Surgery/Society of Thoracic Surgeons position statement on developing clinical practice documents
}

\author{
Faisal G. Bakaeen, MD, ${ }^{\mathrm{a}}$ Lars G. Svensson, MD, PhD, ${ }^{\mathrm{a}}$ John D. Mitchell, MD, ${ }^{\mathrm{b}}$ Shaf Keshavjee, MD, \\ G. Alexander Patterson, MD, ${ }^{\mathrm{d}}$ and Richard D. Weisel, $\mathrm{MD}^{\mathrm{e}}$
}

\footnotetext{
From the a Department of Thoracic and Cardiovascular Surgery, Heart and Vascular Institute, Cleveland Clinic, Cleveland, Ohio; ${ }^{\mathrm{b}}$ Cardiothoracic Surgery Division, University of Colorado Anschutz Medical Center, Aurora, Colo; Divisions of ${ }^{\mathrm{c}}$ Thoracic Surgery and ${ }^{\mathrm{e}}$ Cardiovascular Surgery, Toronto General Hospital, University Health Network and University of Toronto, Toronto, Ontario, Canada; and ${ }^{\mathrm{d}}$ Division of Cardiothoracic Surgery, Washington University School of Medicine, St Louis, Mo.

Disclosures: Authors have nothing to disclose with regard to commercial support.

This article has been co-published with permission in The Journal of Thoracic and Cardiovascular Surgery and The Annals of Thoracic Surgery.

Address for reprints: Faisal G. Bakaeen, MD, 9500 Euclid Ave, Desk J4-1, Cleveland, OH 44195 (E-mail: bakaeef@ccf.org).

J Thorac Cardiovasc Surg 2017;153:999-1005

$0022-5223 / \$ 36.00$

Copyright (c) 2017 by The American Association for Thoracic Surgery and The Society of Thoracic Surgeons http://dx.doi.org/10.1016/j.jtcvs.2017.01.003
}

Improving patient care and achieving better surgical outcomes are key pillars of The American Association for Thoracic Surgery (AATS) and The Society of Thoracic Surgeons (STS) missions. Both organizations have striven over the years to educate cardiovascular and thoracic surgeons about evidence-based practices through various platforms, including the publication of clinical guidelines and consensus documents in their respective journals. In addition, both the STS and AATS have collaborated with other professional associations on scientific publications setting care standards in a multidisciplinary context. The demand for clinical practice guidance has increased in recent years because of an exponential increase in medical knowledge driven by the explosion of new technologies for the diagnosis and treatment of disease.

Medical knowledge continues to grow, and by 2020 a doubling of knowledge is estimated to occur in only 73 days, ${ }^{1}$ increasing the pressure on physicians, surgeons, and other interested parties to keep pace with the boom in knowledge. In addition, patients and payers have come to expect an evidence-based approach in care delivery. Clinical practice guidelines (CPGs) are an option for knowledge to be processed by content experts and disseminated as efficiently as possible in the form of practical recommendations. Noting the wide disparity in the rigor and quality of the processes behind the development of CPGs, a provision in the Medicare Improvements for Patients and Providers Act of 2008 directed the Institute of Medicine (IOM) to develop standards for CPG developers. This culminated in the 2011 IOM report, Clinical Practice Guidelines We Can Trust. ${ }^{2}$

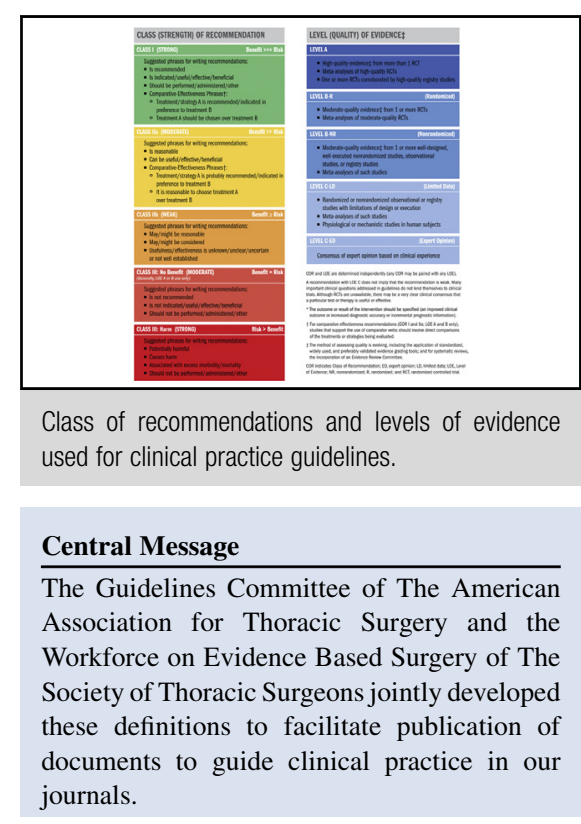

Useful resources (such as the National Guideline Clearinghouse [http://www.guideline.gov]) have emerged to help users identify quality CPGs that adopt many of the principles set forth by the IOM, but compliance with the IOM standards remains difficult, ${ }^{3}$ and some CPG developers are of the opinion that they are more aspirational and not entirely attainable. ${ }^{4}$

Thus, approaches toward clinical practice documents have taken varying formats, such as those espoused by the IOM, ${ }^{2,5,6}$ the Consensus Guidelines proposed by AATS, consensus statements from an invited group of experts as has been done on some topics by STS, ${ }^{8}$ position statements, ${ }^{9}$ and white papers. ${ }^{10}$

STS and AATS have increasingly adopted many of the IOM principles to ensure rigorous and transparent evaluation of current evidence relating to the timely and appropriate management of cardiovascular and thoracic diseases. This work has been conducted by experts in the field organized and supervised in recent years by the Workforce on Evidence Based Surgery (WFEBS) and the Guidelines Committee (GC) of the STS and AATS, respectively. Both the WFEBS and the GC have followed their respective rules and procedures for the synthesis of evidence to guide clinical practice. These rules and 
procedures varied, and the need for modernization, standardization, and a uniform process for developing CPGs was declared a priority by the leadership of both the AATS and STS.

The present project involved a collective effort by the AATS and STS to assemble a joint panel of experts to review the available literature on CPGs and existing organizational policies, establish clear definitions relating to the types of published documents, and set rigorous and practical standards that will ensure consistency and quality of all clinical practice documents published by both organizations. We propose a scientific framework for a reliable and transparent synthesis of evidence-based recommendations in cardiothoracic surgery that will ultimately translate into safe and effective care.

\section{METHODS}

The expert panel was composed of participants identified by an initial core group of the chair and immediate past chair of the STS WFEBS (F.B. and J.M., respectively), the cochairs of the AATS GC (L.S. and S.K.), the editor of The Annals of Thoracic Surgery (The Annals) (G.A.P.), and the editor of The Journal of Thoracic and Cardiovascular Surgery (JTCVS) (R.W.). The project and composition of the panel were endorsed by the 2 organizations. The draft report written by the chairs of the WFEBS and GC were distributed to the entire expert panel, and comments solicited via frequent conference calls and online communication.

\section{TYPES OF CLINICAL PRACTICE DOCUMENTS: CPGS VERSUS EXPERT CONSENSUS DOCUMENTS VERSUS EXPERT OPINION/WHITE PAPERS}

Technically oriented topics relating to the type, extent, and conduct of a surgical intervention are of particular interest to the membership of both organizations. CPGs often address the broader scope of indications and timing of treatment, with less emphasis on technical surgical considerations. AATS and STS clinical practice documents intend to fill this need with the appropriate document type based on the level of evidence available and document development process.

There is broad agreement that CPGs should be based on rigorous evidence. However, high-quality randomized studies are often lacking in the surgical literature. Nevertheless, this lack of randomized studies does not necessarily preclude development of CPGs. Well-designed prospective cohort studies, or large registry studies that compare 2 interventions, can result in useful recommendations.

In the surgical field, much of the published literature is based on single-center, noncomparative case series. Higher-quality evidence may never be obtained in certain areas, but lower-level data and case series may still provide opportunities to optimize outcomes that address important and often common clinical questions. In such scenarios it may be appropriate for the expert panel to use their best judgments to make specific and unambiguous consensus statements designed to reduce poor outcomes. The consensus of a diverse group of experts can provide enormous value in these areas with little to no comparative evidence, yet unacceptably and unnecessarily high risk of mortality, major morbidity, or resource use persists.

In recognition of the challenges outlined here and the specific needs of the AATS and STS membership, this panel recommends that clinical practice documents be categorized into 3 types: CPGs developed according to core IOM standards with an a priori literature search performed, registration on the www.guidelines.gov Web site, and the inclusion of at least some Level A or B evidence; expert consensus documents (ECDs); and expert opinion/white papers (Figure 1).

The decision on the designation of a document as a CPG or ECD rests with the WFEBS and GC based on whether a systematic review of comparative data can be performed for most clinical questions that make up a particular topic. If a systematic review is not feasible, yet the WFEBS or GC believe that opportunities to correct major gaps in care exist without direct comparative evidence (and the organizations' governing bodies agree), then an ECD is suitable. A low level of evidence is not sufficient on its own to justify development of an ECD. A gap in care and clear clinical question(s) also should be identified.

ECDs result in statements that are considered clinical "suggestions" rather than outright recommendations and are clearly labeled as such. In addition, unlike CPGs, there is no requirement for grading ECDs with the American College of Cardiology (ACC)/American Heart Association (AHA) class of recommendation or level of evidence designation.

An expert opinion/white paper is a document written by field experts and thought leaders on issues related to new procedures, technologies, or health policy for which there are few data and significant uncertainty. These papers are intended to review the literature and identify conflicting opinions and alternative treatment strategies without making recommendations for practice.

\section{Development Process for Clinical Practice \\ Documents (Figure 2)}

Identifying a relevant clinical topic. A successful clinical practice document starts with an explicit and well-defined reason relating to the prevention, screening, diagnosis, treatment, or follow-up of a disease or condition. The topic and the associated primary question(s) have to be timely and relevant to contemporary practice. Typically, clinical practice documents focus on topics for which there is significant variation in practice, with disparities in associated outcomes. A CPG, ECD, or white paper may also serve the important purpose of stimulating further research.

Writing committee composition. The writing committee for any STS and/or AATS clinical document should be 


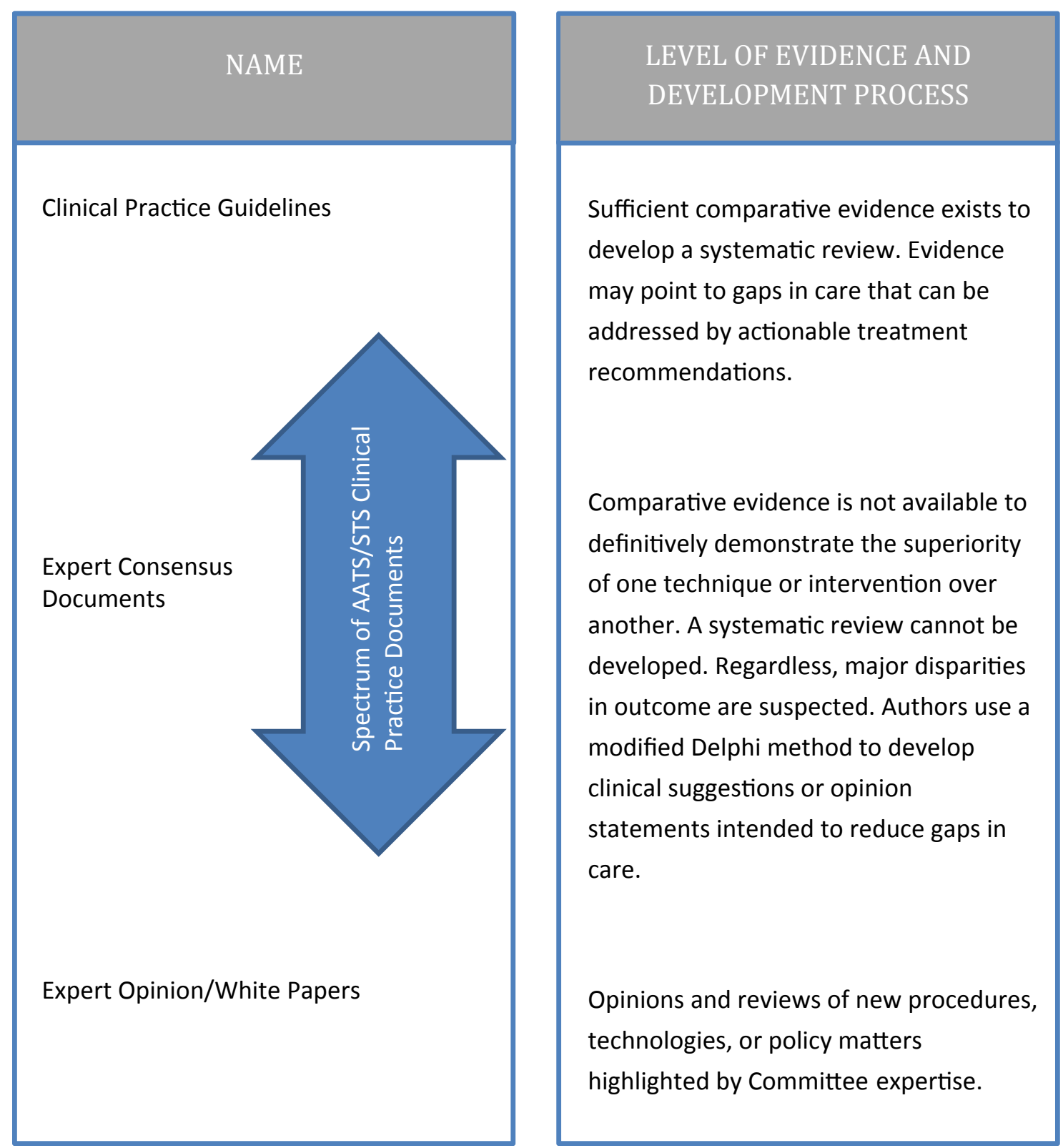

FIGURE 1. Characteristics of various types of clinical practice documents.

made up of published experts on the clinical topic, as well as individuals with experience in CPG development, evidence-based medicine, research, preparing systematic reviews, statistics, epidemiology, and/or quality improvement. Institutional and practice-setting diversity and geographical spread should be reflected in the composition of the committee. The chair/cochairs of the writing committee have primary responsibility for the selection of committee members, development and timely completion of the CPG document, and task distribution among the committee members. All members should contribute substantively to the development of the manuscript, although this contribution can take many forms (eg, helping to shape the patient intervention comparison outcome question if indicated, reviewing evidence tables, drafting a section of the guideline, revising drafts, and/or participating actively in committee calls). Conflicts of interest, relationships with industry, and transparency. The chair(s) and a majority of the authors should be free of significant conflicts of interest. Transparency about relationships with industry should be ensured. Additionally, CPGs, ECDs, and white papers should refrain from recommending specific products or brand names. A committee led by the chair of the WFEBS/cochairs of the GC or their designees will be designated to review these relationships and to certify that undue bias was not 
Reason or Question

Identified and/or approved by AATS/STS Leadership and the AATS GC and STS WFEBS
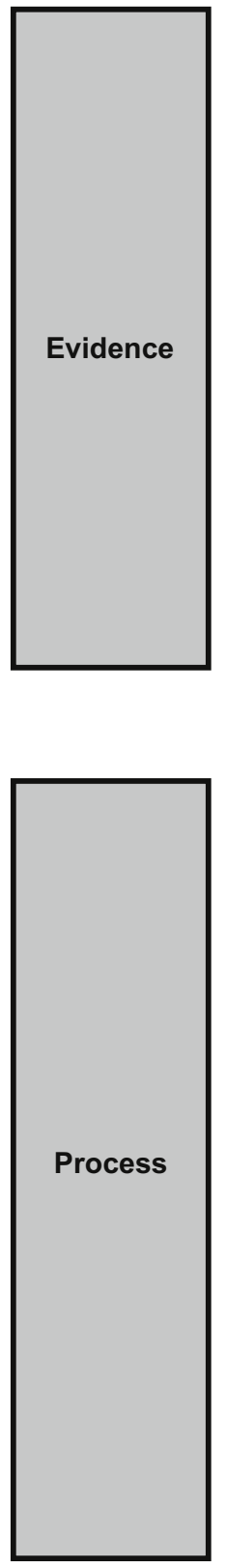

Document development process modeled on IOM standards

Writing Committee may vary, but should incorporate clinical and guidelines development expertise with diverse institutional and geographic representation Robust consensus-building mechanisms

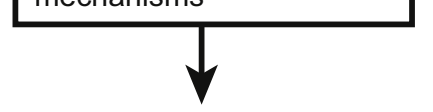

Public comment

ACC/AHA style class of recommendations and level of evidence
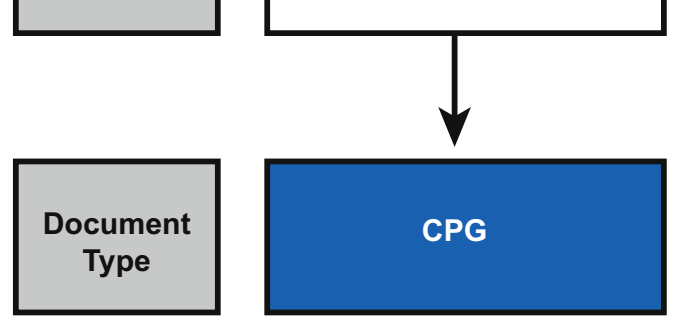

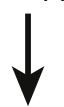

appraisal of evidence

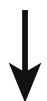

Sufficient comparative evidence

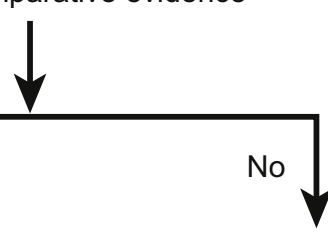

Strong suspicion or
concrete evidence
of a gap in care

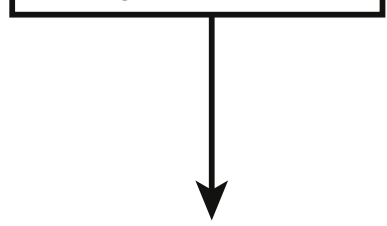

Document primarily developed to summarize various treatment options.

Clinical suggestions suitable in areas where mortality/morbidity/use of resources is unacceptably and avoidably high

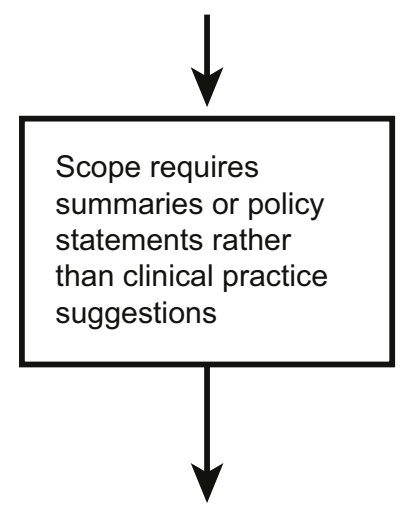

Very limited data, new information requiring timely input by experts regarding potential matters of policy or summarization of a fast-moving field

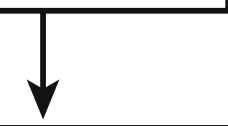

Expert panel organized and sanctioned by AATS/STS Leadership rendering opinion or formulating policy. Transparency and conflict of interest management assured

FIGURE 2. Document pathway: Development process for the 3 types of documents. AATS, The American Association for Thoracic Surgery; STS, The Society of Thoracic Surgeons; GC, Guidelines Committee; WFEBS, Workforce on Evidence Based Surgery; IOM, Institute of Medicine; ACC, American College of Cardiology; $A H A$, American Heart Association; $C P G$, clinical practice guidelines. 

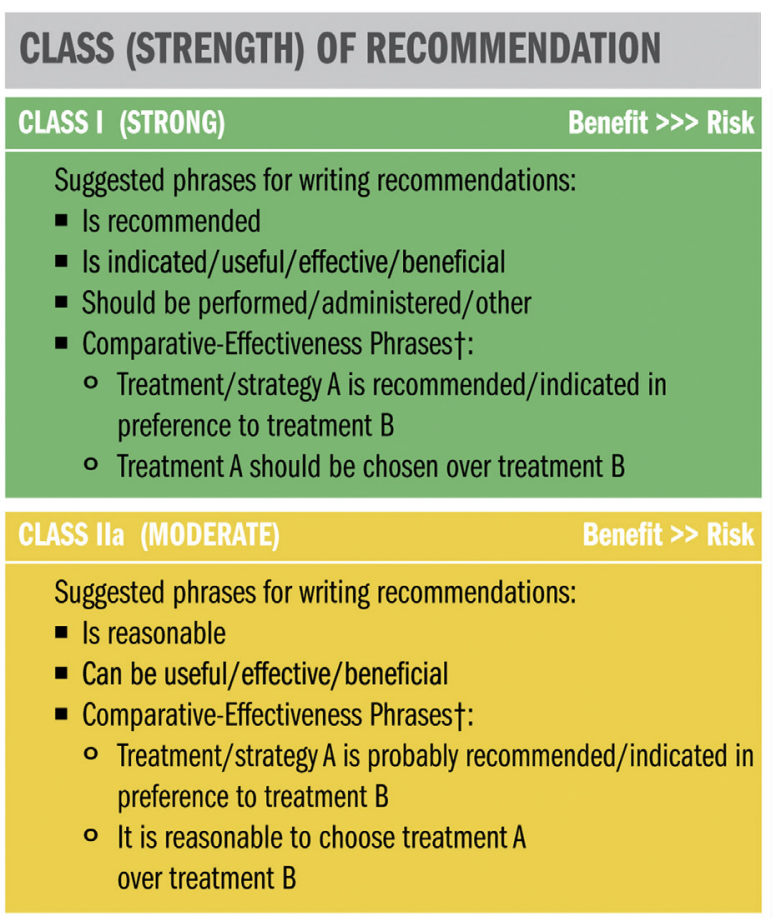

CLASS Ilb (WEAK)
Suggested phrases for writing recommendations:
- May/might be reasonable
- May/might be considered
- Usefulness/effectiveness is unknown/unclear/uncertain
or not well established

\begin{tabular}{l} 
CLASS III: No Benefit (MODERATE) Benefit = Risk \\
(Generally, LOE A or B use only) \\
\hline Suggested phrases for writing recommendations: \\
- Is not recommended \\
- Is not indicated/useful/effective/beneficial \\
- Should not be performed/administered/other
\end{tabular}

\begin{tabular}{ll}
\hline CLASS III: Harm (STRONG) & Risk > Benefit \\
\hline Suggested phrases for writing recommendations: \\
- Potentially harmful \\
- Causes harm \\
- Associated with excess morbidity/mortality \\
- Should not be performed/administered/other
\end{tabular}

\begin{tabular}{l} 
LEVEL (QUALITY) OF EVIDENCE¥ \\
LEVEL A \\
\hline - High-quality evidenceł from more than 1 RCT \\
- Meta-analyses of high-quality RCTs \\
- One or more RCTs corroborated by high-quality registry studies \\
LEVEL B-R \\
- Moderate-quality evidenceł from 1 or more RCTs \\
- Meta-analyses of moderate-quality RCTs
\end{tabular}

LEVEL B-NR
- Moderate-quality evidenceł from 1 or more well-designed,
well-executed nonrandomized studies, observational
studies, or registry studies
- Meta-analyses of such studies

LEVIEI C-LD

(Limited Data)

- Randomized or nonrandomized observational or registry studies with limitations of design or execution

- Meta-analyses of such studies

- Physiological or mechanistic studies in human subjects

LEVEL C-EO N (Expert Opinion)

Consensus of expert opinion based on clinical experience

COR and LOE are determined independently (any COR may be paired with any LOE).

A recommendation with $L O E C$ does not imply that the recommendation is weak. Many important clinical questions addressed in guidelines do not lend themselves to clinical trials. Although RCTs are unavailable, there may be a very clear clinical consensus that a particular test or therapy is useful or effective.

* The outcome or result of the intervention should be specified (an improved clinical outcome or increased diagnostic accuracy or incremental prognostic information).

† For comparative-effectiveness recommendations (COR I and Ila; LOE A and B only), studies that support the use of comparator verbs should involve direct comparisons of the treatments or strategies being evaluated.

$\ddagger$ The method of assessing quality is evolving, including the application of standardized, widely used, and preferably validated evidence grading tools; and for systematic reviews, the incorporation of an Evidence Review Committee.

COR indicates Class of Recommendation; EO, expert opinion; LD, limited data; LOE, Level of Evidence; NR, nonrandomized; R, randomized; and RCT, randomized controlled trial.

FIGURE 3. Updated American College of Cardiology/American Heart Association table of class of recommendation $(C O R)$ and level of evidence ( $L O E)$. $R C T$, Randomized controlled trial. Reprinted with permission from: Jacobs AK, Anderson JL, Halperin JL. The evolution and future of ACC/AHA clinical practice guidelines: a 30-year journey: a report of the American College of Cardiology/American Heart Association Task Force on Practice Guidelines. J Am Coll Cardiol. 2014;64:1373-84.

employed in voting for or against any recommendations or opinion statements within the document. Disclosure forms must be submitted by all members of the writing committee at the beginning of the development process and should be added to the executive summary and to the full-length document. In addition, The Annals and JTCVS will require a separate disclosure form to be signed by all authors at the time of publication. A table containing writing committee member names and their relevant relationships with industry and other conflict of interest information will be included in the supporting materials published by the journals and the organizations' Web sites. 
Literature review and gathering of evidence. After a suitable topic has been selected, the writing committee is responsible for formulating objectives, focused clinical questions using the patient intervention comparison outcome format (PICO), and determining the inclusion and exclusion criteria for relevant publications. At least 2 members of the committee should screen the abstracts of the search results for relevance.

The literature search should be systematic and documented with reproducible methodology that allows others to duplicate it. The search strategy is posted on the STS and AATS Web sites and as an online appendix for the journals as supporting material for the document. If the systematic search does not identify enough published evidence to support writing a CPG, the authors may choose to write an ECD instead.

For CPGs, the available literature will be gathered, and if relevant, STS/AATS staff will work with the writing committee to develop fields for an evidence table. These may include information about study design, sample size, and specific outcomes data chosen before data analysis. Additional tables will be developed to assess the individual quality of each paper and its risk of bias. A different grading scale is used for each type of study and may include Jadad for randomized controlled trials, Newcastle Ottawa for observational studies, and Quality Assessment of Diagnostic Accuracy Studies (QUADAS)-2 for diagnostic studies.

Because a systematic review is in essence a method to determine superiority of 1 intervention over another, an evidence table and quality assessment tables are not applicable if direct comparative data are lacking. An ECD will often rely extensively on a single-arm case series for which a systematic review is not appropriate, and expert opinion and experience is the primary factor in estimating an overall effect of an intervention. The resulting manuscript should prioritize summarizing various options, listing pros and cons of each approach, and making clinical suggestions only in the situation where comparative evidence is not necessary to reduce poor outcomes.

Evidence appraisal for CPGs. Evidence derived from the literature review and evidence tables is often heterogeneous in regard to study design, risk of bias, and reporting of outcomes. The writing committee is tasked with distilling the data, study quality assessments, clinical judgments, and patient preferences into CPG recommendations. The writing committee should provide clear and explicit documentation of how these elements led to the recommendations.

STS and AATS use the updated table "Classification of Recommendations and Level of Evidence" published by ACC/AHA (Figure 3), as well as the phrases for writing recommendations suggested by the ACC/AHA. ${ }^{5}$ It is important that the recommendations be grammatically correct, actionable, and avoid ambiguous or vague language.

Consensus for CPGs. Recommendations will be developed using a modified Delphi method.

1. Potential recommendations are drafted via conference call or a face-to-face meeting.

a. All potential statements will be included in an electronic survey using a 5-point Likert scale (where $1=$ strongly disagree, $2=$ disagree, and so on) to determine inclusion in the final draft.

b. Once an $80 \%$ response rate is achieved, statements in which $75 \%$ of respondents "agree" or "strongly agree" proceed to the next step.

2. An electronic survey is circulated with multiple choice options for assigning a class and level of evidence for each recommendation. An $80 \%$ response rate, with a minimum of $75 \%$ agreement on class and level, is required for consensus.

a. The chair/cochairs are responsible for facilitating exchange and discussion among the authors, resolving any differences in class and level for each recommendation, and selecting precise wording of each recommendation. A second or third round of voting may take place to reach the $75 \%$ threshold for any class and level that was not agreed upon in prior rounds.

b. In the unlikely scenario of enduring differences, there will be an opportunity for writing committee members to add commentary notes concerning a recommendation that the committee was unable to gain consensus on and to express their views as to whether further research is needed to resolve the issue.

c. Members with significant conflicts of interest should be recused from voting on specific recommendations relevant to that conflict.

It is important to refrain from making any recommendations when there is insufficient evidence or a split in the opinion of experts. Such scenarios carry a substantial risk that the recommendation may be wrong. In such situations, research recommendations should be suggested.

Consensus for ECDs. The consensus development process for ECDs follows a similar modified Delphi method. However, the ACC/AHA classification system assignments are not required. Multiple rounds of Likert scale voting may proceed if statements do not achieve a $75 \%$ "agree" or "strongly agree" response after the first round.

Review, approval, and endorsement process. Once the draft document is approved by a writing committee, it will be submitted to the WFEBS and GC for review and 
comment. In the case of partnering societies, a draft will be forwarded to the organizations and circulated according to their processes. All comments must be received within 2 weeks. Additional internal review and approval by higher governing bodies will follow before the draft is posted online by the STS, AATS, and partnering organizations for a 2-week comment period by the relevant membership(s).

Based on comments received in response to the posting, the writing committee, working in conjunction with the WFEBS and GC chairs, will modify the document as deemed appropriate. Any changes to the evidence and recommendations made during the approval process must go back to the authors and the WFEBS/GC chairs for review and verification of evidence. The document is then forwarded to higher governing bodies for final approval and submission to The Annals and JTCVS.

Distribution. Currently, CPG distribution includes publication in The Annals and JTCVS and posting on the STS and AATS Web sites. CPGs will also be available on other online and mobile platforms, including organization iPhone applications, downloadable through the iTunes store. All CPGs will be submitted to the National Guideline Clearinghouse for consideration for posting on its site. Partnering or collaborating societies may also distribute CPGs. CPGs also may be presented in e-learning modules for continuing medical education.

ECDs and white papers will be published in The Annals, $J T C V S$, and the journal of any partnering or collaborating society.

\section{CLINICAL PRACTICE GUIDELINE UPDATE POLICY}

In keeping with the National Guideline Clearinghouse requirements, a process should be in place to ensure that all CPGs are updated at least every 5 years to evaluate the influence of new research on recommendations contained within the original publication. This may take the form of only updating sections for which there is new and relevant information related to previous recommendations, as is done by AHA/ACC. ${ }^{5}$ It is recommended that the writing committee conduct a routine review yearly or when there are new and important studies relevant to the CPG. A CPG and its recommendations are considered to remain valid if results from postpublication clinical studies do not contradict earlier research results.

Barring any drastic changes to medical consensus, an update involves a new literature search and an addendum to the prior publication outlining important studies within the field that may or may not make slight changes to levels of evidence and strength of recommendations.

\section{References}

1. Densen P. Challenges and opportunities facing medical education. Trans Am Clin Climatol Assoc. 2011;122:48-58.

2. Institute of Medicine. Clinical practice guidelines we can trust. Washington, DC: The National Academies Press; 2011.

3. Kung J, Miller RR, Mackowiak PA. Failure of clinical practice guidelines to meet Institute of Medicine standards: two more decades of little, if any, progress. Arch Intern Med. 2012;172:1628-33.

4. Qaseem A, Forland F, Macbeth F, Ollenschlager G, Phillips S, van der Wees P, et al. Guidelines International Network: toward international standards for clinical practice guidelines. Ann Intern Med. 2012;156:525-31.

5. Jacobs AK, Anderson JL, Halperin JL, Anderson JL, Halperin JL, Albert NM, et al; ACC/AHA Task Force Members. The evolution and future of ACC/AHA clinical practice guidelines: a 30-year journey: a report of the American College of Cardiology/American Heart Association Task Force on Practice Guidelines. Circulation. 2014;130:1208-17.

6. Aldea GS, Bakaeen FG, Pal J, Fremes S, Head SJ, Sabik J, et al. The Society of Thoracic Surgeons Clinical Practice Guidelines on Arterial Conduits for Coronary Artery Bypass Grafting. Ann Thorac Surg. 2016; 101:801-9.

7. Svensson LG, Gillinov AM, Weisel RD, Keshavjee S, Bacha EA, Moon MR, et al. The American Association for Thoracic Surgery Consensus Guidelines: reasons and purpose. J Thorac Cardiovasc Surg. 2016;151:935-9.e1.

8. Svensson LG, Kouchoukos NT, Miller DC, Bavaria JE, Coselli JS, Curi MA, et al. Expert consensus document on the treatment of descending thoracic aortic disease using endovascular stent-grafts. Ann Thorac Surg. 2008;85(1 Suppl): S1-41.

9. Vassiliades TA Jr, Block PC, Cohn LH, Adams DH, Borer JS, Feldman T, et al. The clinical development of percutaneous heart valve technology: a position statement of the Society of Thoracic Surgeons (STS), The American Association for Thoracic Surgery (AATS), and the Society for Cardiovascular Angiography and Interventions (SCAI) Endorsed by the American College of Cardiology Foundation (ACCF) and the American Heart Association (AHA). J Am Coll Cardiol. 2005;45:1554-60.

10. Kauczor HU, Bonomo L, Gaga M, Nackaerts K, Peled N, Prokop M, et al ESR/ERS white paper on lung cancer screening. Eur Respir J. 2015;46: 28-39. 